\title{
Distribución espacial de la pobreza Distrito Federal de México 1990-2040
}

\author{
Gardy Augusto Bolívar Espinoza \\ Universidad Autónoma de México - Sede Azcapotzalco, Ciudad de México, México. \\ Email: abe88@hotmail.com
}

\section{Óscar Rogelio Caloca Osorio}

Universidad Nacional Autónoma de México, Ciudad de México, México.

Email: oscarcalo8@yahoo.com.mx

La búsqueda de la felicidad exige un compromiso activo con las tareas vitales, que implican placer en el despliegue de capacidades y conocimientos. (...). La pobreza, como otras circunstancias vitales potencialmente debilitadoras, puede ser opresiva porque puede conducir a la disminución de dichos conocimientos y capacidades y porque puede provocar una atmósfera de desesperación

(Giddens; 1998: 198).

Resumen: A partir de una discusión sobre el concepto de pobreza desde la perspectiva de las necesidades, absoluta y relativa, y su satisfacción, se exponen estrategias metodológicas y alternativas para su medición. Considerando zonas espaciales, se estima la pobreza territorial para las delegaciones del Distrito Federal sincrónica y diacrónicamente en el periodo 2000-2040. Se hace uso del método de ESNBI a fin de comparar espacios territoriales demarcados por una división política, lo cual se desarrolla a través de variables de Necesidades Básicas o variables directas y un indicador de feminización de la pobreza. A las variables seleccionadas se les da un tratamiento con base en el Método de Componentes Principales que da como resultado un índice de pobreza.

Palabras clave: Pobreza, capacidades mermadas, análisis espacial.

\section{Spatial distribution of poverty. Federal District of Mexico 1990-2040}

\begin{abstract}
After a discussion of the concept of poverty from the perspective of the needs, absolute and relative, and its satisfaction, are presented here alternatives and methodological strategies for its measurement. Considering space areas for the territorial delegations of the Federal District, poverty is estimated synchronically and diachronically in the period 2000-2040. The method ESNBI is used in order to compare territorial spaces demarcated by a political division, which is developed through variables of basic needs or direct variables and an indicator of the feminization of poverty. The variables selected are given a treatment based on the Main Components Method resulting in a poverty index.
\end{abstract}


Key words: Poverty, diminished ability, spatial analysis.

\section{Distribuição espacial da pobreza. Distrito Federal do México 1990-2040}

Resumo: Depois de uma discussão sobre o conceito de pobreza a partir da perspectiva das necessidades, absolutas e relativas, e sua satisfação são apresentadas alternativas e estratégias metodológicas para a medição. Considerando as áreas do espaço, a pobreza é estimada para as delegações territoriais do Distrito Federal sincronicamente e diacronicamente, no período 2000-2040. Ele usa o método ESNBI para comparar os espaços territoriais demarcados por uma divisão política, que se desenvolve através de variáveis de necessidades básicas ou variáveis diretas e um indicador da feminização da pobreza. As variáveis selecionadas recebem um tratamento baseado no método de componentes principais, resultando em um índice de pobreza.

Palavras-chave: Pobreza, uma capacidade diminuída, análise espacia.

$$
* * *
$$

\section{Introducción}

En el siglo XX se impone el carácter controversial de la relación entre mercado y Estado, discusión teórica ampliamente formalizada. Desde lejanas épocas el capitalismo tiende a ser hegemónico en el mundo dejando atrás esquemas tradicionales de reproducción y superando posturas rígidas del socialismo. Bajo imperativos técnicos y de competencia afecta en forma significativa las esferas de la política y de lo social constituyendo un mundo moderno cada vez más distante del antiguo régimen como de las formas locales de convivencia. La dinámica del capital y su modelo tienen un impacto insoslayable en la vida de las naciones, tanto en la producción de bienes y servicios, el empleo y el consumo como en los procesos de urbanización y el crecimiento de las ciudades.

Bajo nuevos auspicios en este complejo proceso de mundialización, que involucra formas diversas de creencias, culturas, modos de ser, sentir y actuar, se ha repuesto la discusión expuesta ejemplarmente y originariamente por Condorcet, sobre la idea del progreso y el bienestar así como la elección social razonada para hacerla efectiva. Hoy, se manifiesta en la discusión formalizada en torno al Social Choice y al Welfare iniciadas por Kenneth Arrow, Amartya Sen y John Rawls para mencionar sólo unos pocos de los brillantes intelectuales que pusieron en el tapete, desde la década de los setenta, en forma integrada y transversal, los temas del bienestar, la justicia y pluralismo.

Entre otras cosas, a partir de ese entonces se discute y argumenta con mayor intensidad, sobre la posibilidad que los fallos del mercado pudieran ser corregidos a través de políticas públicas o, por el contrario, el sistema, por sí mismo, se encargará de otorgar el mejor equilibrio posible 
para la mayoría. Pero, este dilema, no puede superar una porfiada constante que cuestiona el progreso y el bienestar en su aspecto más sensible, la reducción de la pobreza, la inequidad y la injusticia. Si interviene el Estado, la dictadura de las decisiones abstractas y generales no asegura la mejor decisión y, la experiencia muestra, que tampoco resuelve los problemas de justicia social. Si se opta por el óptimo paretiano la concentración de la riqueza persiste en su crecimiento y la distancia entre ricos y pobres se acrecienta cuestionando la libertad y la felicidad que se pretende perseguir.

Las hambrunas laceran más que nunca inmensas regiones del orbe, con distintas intensidades y diversas cualidades. Los grandes avances científico-tecnológicos de los siglos XIX y XX que transformaron radicalmente la vida de los hombres en el planeta y que sugirieron la esperanza de disminuir la pobreza, a pesar de sus logros extraordinarios, no logran resolver situaciones que se consideraban fáciles de abatir: la pobreza. Aparentemente el problema no es técnico si no de justicia y legalidad.

Más que una discusión sobre las condiciones generadoras de pobreza y de las formas de eficaces de atacarla interesa, previamente, describirla en un espacio y en un tiempo determinados con el fin de contribuir a la elaboración de políticas públicas que puedan paliar la pobreza en el futuro. A través del estudio de la situación actual de pobreza que padece el Distrito Federal se proponen algunas hipótesis sobre su situación futura. En este espacio, si bien la pobreza ya no es vista como una fatalidad, se presenta como una merma significativa de las capacidades para lograr la satisfacción de las necesidades básicas. Este trabajo, pretende dar cuenta tanto de la existencia y la distribución espacial como de los cambios en los niveles de pobreza existentes entre las delegaciones del Distrito Federal para el periodo 1990-2040.

En la primera, sección se propone el concepto de pobreza adoptado. En la siguiente se establecen el marco de referencia y la metodología para el manejo de las variables que permita identificar la pobreza en las delegaciones del DF entre 1990-2040. En tercer lugar, se exponen los resultados obtenidos y, en la cuarta sección, se muestra: un ordenamiento espacial del territorio identificado por Zonas Espaciales de Pobreza 1990-2040 (ZEP); su distintiva convergencia en la reducción de los niveles de pobreza y su transformación entre 1990-2040. Por último, en los anexos se expone el método de estimación de componentes principales y algunas otras consideraciones metodológicas.

\section{Pobreza y necesidades}

La pobreza se considera como una situación indeseable que ofende a la sociedad: persistente, difícil de erradicar o, ineludible. Su concepto a veces abarca el campo de la positividad o el de la normatividad. Es decir, desde la aceptación fenomenología de los hechos de la vida social hasta la aspiración y obligación, del deber ser, de su erradicación. En este sentido 
Eric Hobsbawm sostiene que "la pobreza se define siempre de acuerdo con las convenciones de la sociedad donde ella se presente” (Apud. Sen; 1992: 314). Pareciera que la comprensión del concepto de pobreza radica en el marco de las necesidades: cada vez que se les identifica debieran ser satisfechas, al menos, las necesidades que se consideran esenciales, básicas. Esto le confiere a todo concepto de pobreza un marco de referencia en la vida misma y en la explicación de la evolución y adaptación de los individuos a un medio social efectivo. Para esta investigación se considera como apropiado el argumento sobre el carácter histórico de las necesidades: transformaciones sociales e institucionales que se materializan a través de la transformación de las necesidades o la generación de nuevas necesidades.

\section{El campo de las Necesidades}

La indagación acerca de las necesidades presenta diversos ámbitos, pero en particular atienden a la determinación del carácter de las necesidades, es decir, si son verdaderas o falsas (Heller, 1996). La determinación de existencia de falsas necesidades refiere a: la contingencia de satisfacer ciertas necesidades, o, la obligación de satisfacer todo aquellos que aparece como necesidad. En cualquier caso lo relevante es la importancia relativa que se brinda a las necesidades y el enfoque contextual percibido, donde comenzamos..."a vivir con diferentes dotes de riqueza y compromisos heredados. Habitamos en diferentes ambientes naturales, algunos más hostiles que otros. Las sociedades y comunidades de las que formamos parte nos ofrecen diversas oportunidades de lo que podemos o no podemos hacer” (Sen: 1995; 32). Y esto condiciona lo que se considera necesario.

Así, la determinación de las necesidades en general es compleja, puesto que es difícil considerar qué es necesario y qué no lo es, como es el caso de la alimentación adecuada, por ejemplo. Las necesidades no podrían ser determinadas de manera exhaustiva e inamovible siguiendo criterios naturales permanentes, solamente; se requeriría también, considerar algunos patrones de consumo orientados por la regla de la mayoría. Junto a los requerimientos de proteínas, calorías por ejemplo, deberían considerarse los aspectos culturales, los cuales, para efectos de su medición sería necesario traducir en una canasta de alimentos que indudablemente contemple el tipo de dieta de los individuos de un espacio-tiempo determinado.

Lo anterior conduce a la determinación de una canasta de referencia, indudablemente excluyente, que permite establecer una norma mínima de satisfacción de necesidades; la cual puede ser constituida por ley o con base en la percepción de una muestra de individuos representativos que creen que lo contenido en la norma es lo necesario, es decir, se establece una norma intersubjetiva. Este tipo de procedimiento establece que para cada uno de los elementos considerados dentro de la norma, es necesario saber si estas satisfacen una necesidad o sólo si se percibe que satisfacen una necesidad, es decir, se identifica a la necesidad en sí o los que se considera subjetivamente que puede ser la necesidad. 
En la búsqueda de satisfacción de necesidades es relevante la diferencia que existe entre la percepción de las necesidades y las necesidades en sí, porque, si nunca se ha tenido algo en particular ¿cómo saber que ese algo es sólo deseable o es necesario? Tal circunstancia aparece principalmente en situaciones de privación duradera que conducen a una contracción o eliminación de deseos, porque:

en situaciones de privación duradera las víctimas no siguen quejándose y lamentándose todo el tiempo y, muy a menudo, hacen grandes esfuerzos para gozar de los pequeños placeres a su alcance y reducir sus deseos personales a proporciones modestas o "realistas" (...) la razón prudencial aconseja que concentren sus deseos en aquellas cosas limitadas que quizá puedan alcanzar, en vez de aspirar infructuosamente a lo que es inalcanzable (...) incluso si esa persona no está adecuadamente alimentada, decentemente vestida, mínimamente educada y convenientemente alojada (Sen; 1995: 68).

Lo anterior, conduce a que la percepción sobre las necesidades básicas puede no corresponder con los deseos de satisfacción de las mismas, y sí, con una percepción que puede estar por debajo del quantum de satisfacción de necesidades básicas que la sociedad reconoce como mínimas.

En el caso de la alimentación, gran parte de las dificultades que surgen en la elección de los alimentos por satisfacer, que sirven como referencia en la determinación de una canasta alimentaria, corresponden con la propia definición de la canasta alimentaria. Porque, en la mayor parte de los casos la selección de la canasta alimentaria corresponde con una lista inicial de alimentos que se deben incluir. Esta lista normalmente se selecciona con base en los alimentos que representan la mayor parte del gasto alimentario de las familias ${ }^{1}$. Este procedimiento puede objetarse, por tomar la realidad observada como base de la norma, sin precisar si las familias consumen dichos alimentos, porque así lo desean o por limitaciones de recursos (Boltvinik; 1997: 382). Sin embargo, es el mecanismo de referencia empleado en la mayor parte de las investigaciones sobre pobreza que se rigen por una canasta alimentaria.

\section{Privación relativa y absoluta e identificación y agregación}

Una vez llevada a cabo la breve revisión a las necesidades, su alcance y límite; expresado por la percepción de necesidades y no de las necesidades en sí, nos introducimos en la concepción sobre pobreza con base en el enfoque de Privación Relativa² y su relación con la Privación Absoluta.

La circunstancia que un individuo sea pobre refiere a que este tiene una carencia que no puede ser satisfecha. Pero que esta carencia está sujeta al reconocimiento social de que esta situación efectivamente es una carencia que tiene que satisfacerse. Esto se traduce en la existencia de una privación que no corresponde con una percepción de lo necesario y sí con aquello que es necesario satisfacer. 
Tal privación cada vez que ocurre bajo la interacción entre los individuos y con su medio, se define como una privación relativa, porque esta depende del espacio-tiempo, es decir, de donde y cuando ocurre tal situación de privación. La interacción social es determinante para que los individuos consideren que están privados de la satisfacción de una necesidad ${ }^{3}$. Con ello, para todo animal social, el concepto de privación es relativo, porque la interacción social conduce a diferenciar entre sentimientos de privación y condiciones de privación. Los primeros, corresponden con la percepción de las necesidades y las segundas con la insatisfacción de las necesidades en sí ${ }^{4}$.

La categoría de pobreza no sólo requiere de una concepción basada en la privación relativa también tiene que ser una construcción que se forme a través de un núcleo irreductible de necesidades no satisfechas, es decir, tal noción depende también de considerar una privación absoluta de necesidades. En consecuencia, toda categorización de pobreza requiere de un argumento basado en un núcleo irreductible de necesidades por satisfacer para todos los individuos en cualesquier espacio-tiempo y, de una noción que haga referencia al medio-social y cultural particular. En otras palabras, toda categoría de pobreza tiene que integrar por una parte un núcleo irreductible de privación y por otra, la calidad histórico-dinámica que conduce a la transformación y creación de necesidades.

El núcleo irreductible de privación absoluta permite identificar una serie de necesidades que rebasan cualquier espacio-tiempo. Así, aquellos individuos que no logren satisfacer dichas necesidades se encontraran en una situación de privación absoluta y por ende, de pobreza. En el caso de la privación relativa, los grupos sociales de mayor vulnerabilidad o que han experimentado marginación social y/o se les ha relegado a través de la discriminación ya sea por diferencias de sexo, raza, comunidad y/o clase, presentan privaciones relativas que difícilmente pueden ser evaluadas debido a la contracción de sus deseos. Sin embargo, estos, al manifestar una privación relativa se encuentran también en una situación de pobreza.

Por otra parte, toda categoría de pobreza debe contemplar la integración de dos cuestiones más: la identificación y la agregación. La primera refiere a la ubicación de un grupo de personas en la categoría de pobres, y la segunda, a la posibilidad de unir las características del conjunto de pobres en una imagen global de pobreza. Con ello, se establece quiénes son pobres y qué se considera una situación de pobreza.

La determinación de quiénes son pobres consiste en definir un conjunto de necesidades básicas o mínimas que deben ser satisfechas. La segunda, corresponde con la noción de capacidad para satisfacer dichas necesidades donde, esta incapacidad, es la prueba de pobreza, pues los individuos en general difieren entre si; $y$, son estas diferencias, las que median sobre las oportunidades que se tienen para la satisfacción de necesidades. En este caso no se puede descartar que el nivel de ingresos es importante 
"si se considera un caso de reducción real del ingreso y un incremento del sufrimiento de todos los pobres, ello tendrá que describirse como un aumento de la pobreza (...) El foco del concepto de pobreza tiene que ser el bienestar de los pobres como tales, sin importar los factores que lo afecten.” (Sen: 1992; 311).

La noción de capacidad vista como la viabilidad para satisfacer las necesidades básicas o mínimas, depende de lo que se espera sea el bienestar de una persona con base en su vida. La vida puede ser vista como un conjunto de funcionamientos y la realización de esta como una selección particular del conjunto de funcionamientos; entendiendo por funcionamientos aquellas cuestiones que pueden abarcar desde el estar suficientemente alimentado, tener buena salud, evitar posibles enfermedades y mortalidad prematura, hasta actividades vitales más complejas como el ser feliz, el tener dignidad, el participar en la vida en la comunidad (Sen; 1995: 53). En este sentido, la capacidad o las capacidades de los individuos pueden entenderse como la viabilidad de alcanzar un conjunto particular del total de posibles combinaciones de funcionamientos.

Por ende, que un individuo o grupo de individuos sea considerado o no pobre dependerá de la capacidad que este tenga para alcanzar un cierto nivel de bienestar. Esto trae consigo que mientras la libertad o las oportunidades de un individuo o grupo de individuos, para lograr el nivel de bienestar mínimo esperado, se vean mermadas, su capacidad para alcanzar los funcionamientos elegidos disminuye, con ello, aumenta la privación para la satisfacción de necesidades, y por ende, este o estos individuos serán pobres.

Así, de no existir libertad y oportunidades para que los individuos alcancen los funcionamientos elegidos como mínimos, su capacidad para tener bienestar será mermada en grado tal que traerá consigo la privación absoluta y relativa de satisfacción de necesidades. En este sentido, de acuerdo con lo anterior para nosotros pobreza es: la falta de libertad y oportunidades que merman la capacidad para alcanzar los funcionamientos elegidos como mínimos, según el contexto, y que trae como consecuencia la reducción del bienestar de los individuos debido a una privación absoluta y relativa de satisfacción de necesidades.

\section{Medición de la pobreza}

Una vez que se cuenta con un concepto de pobreza se requiere atender la medición encaminada a la identificación de zonas de pobreza, en este caso, la unidad de análisis es la delegación del DF. Es así como se discute acerca de las principales maneras de estimar la pobreza y cuál de estas es la conveniente dada la estructura de la investigación y la unidad de análisis.

La pobreza se puede medir con indicadores directos de insatisfacción de necesidades o con un indicador indirecto: el ingreso. Este 
suele ser empleado como un indicador esencial, empero con él, no se mide la satisfacción de alguna necesidad o grupo de necesidades, más bien se mide únicamente la capacidad del hogar para satisfacer necesidades. Es por ello que se le conoce como el método indirecto de medición de la pobreza. Sin embargo, existen métodos directos que corresponden con el hecho de que se cuente o no con el factor determinado.

Pero los estudios sobre la pobreza y su derivación hacia el tema de la desigualdad, puede decirse, que se desarrollan en torno al excelente trabajo sobre la medición y posteriormente la profundización hacia el tema de las capacidades y de la libertad inaugurado por Amartya Sen, Rawls y Arrow ${ }^{5}$. En este entorno teórico, a la altura de los noventa, la discusión principal, se separa claramente de la perspectiva del crecimiento económico hacia dominios más sociológicos pero liderados por la económica: “el imperialismo de la teoría económica”6. En este medio se acuña el concepto de capital social al mismo tiempo que se desarrolla en Europa y en especial con la obra de Pierre Bourdieu.

Existen diversos métodos para la medición de la pobreza, de los cuales destacan el Enfoque Sectorial de Necesidades Básicas Insatisfechas (ESNBI), el método de Necesidades Básicas Insatisfechas (NBI), método de Línea de Pobreza (LP) y el Método de Medición Integrado de la Pobreza (MMIP) (Boltvitnik y Hernández Laos; 1999: 45).

El Enfoque Sectorial de Necesidades Básicas Insatisfechas, consiste en definir un mínimo de cada necesidad y calcular la población por debajo de cada una de ellas. Con ello todos aquellos que no satisfacen la necesidad se encuentran en situación de pobreza. Otra forma de evaluar el fenómeno corresponde al Método de Necesidades Básicas Insatisfechas, en este se selecciona un conjunto de necesidades y se define un número de indicadores para su cumplimiento, en este se trabaja con hogares, lo cual permite identificar hogares y personas pobres, que se encuentran por debajo del nivel mínimo del cual se considera satisfecha la necesidad.

Por su parte, el método de la Línea de Pobreza se forma a partir de considerar, de manera indirecta, una canasta de bienes y servicios cuyo costo se compara con el ingreso per cápita (Olson, 1965) pobres. Sin embargo, "la magnitud de la brecha de los ingresos de los pobres con respecto a la línea de pobreza: una reducción de los ingresos de todos los pobres, sin afectar los ingresos de los ricos, no modificará en absoluto la tasa de incidencia. En segundo lugar, es insensible a la distribución del ingreso entre los pobres” (Sen: 1992; 311). Esto hace pensar que la determinación de la pobreza a través de este único método es cuestionable. Sin embargo, de la unión de los dos últimos enfoques se desprende el Método de Medición Integrada de la Pobreza. En este se considera, por hogares, la relación existente entre el método de Línea de Pobreza aunado al Método de NBI del cual se selecciona un determinado número de variables, dando como resultado un reporte que corres- 
ponde con indicadores directos (NBI) e indirectos (LP). Por último, se tiene la existencia de otro método que pretende medir la cantidad y calidad de vida, al cual se le conoce como el Método de Medición de la Cantidad y Calidad de Vida, en este se incluye una medición sobre la duración de la vida o esperanza de vida al nacer.

De acuerdo con Hernández Laos, conviene señalar que en el presente, los métodos a los que más recurren los investigadores para analizar la pobreza con base en los hogares son el NBI, LP y MMIP. Donde, el empleado para la medición de la pobreza en territorios particulares es el de ESNBI.

\section{Estrategia}

En el presente trabajo se hace uso del método de ESNBI puesto que se pretende comparar espacios territoriales demarcados por una división política, lo cual se desarrolla a través de variables de Necesidades Básicas o variables directas y un indicador de feminización de la pobreza ${ }^{7}$. Pero con la variante de que a las variables seleccionadas se les da un tratamiento con base en el Método de Componentes Principales (véase Anexo), que da como resultado un índice de pobreza.

La elección de las variables corresponde con un conjunto particular, este se considera como el de mayor grado explicativo para dar cuenta de la existencia de pobreza o no en un territorio en particular. Así, con base en la unidad de análisis y tomando en cuenta el proceso seguido por los asentamientos humanos. Las variables se agrupan en tres conjuntos generales: 1) variables de vivienda, 2) educación y 3) factor de feminización de la pobreza.

En el caso de la vivienda, se considera que este es un indicador fundamental porque media la espacialización de la pobreza, esto es, la localización en el territorio de viviendas que por sus condiciones y/o carencia de servicios se infiera que pertenecen a familias pobres. Esto visto en el contexto del DF, es relevante debido a que en el estudio de la pobreza no es la carencia de vivienda propia o rentada lo que se requiere identificar y sí lo es las condiciones y servicios existentes en dichas viviendas. Donde los pobres del DF se caracterizan principalmente por contar con viviendas insuficientes o de mala calidad: a diferencia de otras grandes ciudades, en el DF es mínima la población que duerme en las calles.

Asimismo, en el DF existe una gran heterogeneidad en la vivienda de los pobres, que es consecuencia de la diferencia de oportunidades y situaciones que enfrentan los pobres para hacerse de una vivienda. Lo anterior es relevante cada vez que la vivienda expresa las diferencias de la producción social en el espacio modelando las condiciones de vida del grupo doméstico. Porque muchos de los pobres del DF se localizan en zonas de riesgo o en asentamientos irregulares. 


\section{Segregación socio-espacial: asentamientos populares 1990-2000}

Una vez realizadas las estimaciones pertinentes para 1990 y 2000 se encontró que la propia dinámica del crecimiento urbano, debida al crecimiento de los asentamientos humanos y a la localización de estos, refuerza la idea sobre la existencia de un proceso de segregación socioespacial, cuya representación se expresa principalmente en la conformación de asentamientos populares, en amplios segmentos del territorio.

Esto puede ser visto a partir de la agrupación de los resultados en cinco niveles de observación, que corresponden con una determinada proporción de pobreza existente entre las delegaciones del DF. En primer lugar se cuenta con un nivel de muy baja pobreza el cual indica que en las delegaciones seleccionadas con esta característica los niveles de pobreza son bajos, es decir, si bien existen pobres estos no son un número importante, posteriormente existen los niveles de baja, media y alta pobreza, para finalizar en el nivel de muy alta pobreza, el cual indica que el número de pobres que se localizan en tales delegaciones es cuantioso y el mayor respecto de cualquier delegación que se ubique en los otros cuatro niveles.

Esto no debe ser interpretado como que todo el DF es pobre y sí como el hecho de que en todas y cada una de las delegaciones existen diferentes cuantías de pobres: en algunas menos y en otras más; como en el nivel de alta y muy alta pobreza. Asimismo, las delegaciones pueden ser clasificadas según su incorporación al proceso de urbanización de la Ciudad de México, tal proceso se conoce como los contornos del DF: La Ciudad Central y tres contornos.

A) La Ciudad Central. Corresponde con el territorio que abarcaba la Ciudad de México en 1930 y actualmente se configura por medio de las delegaciones: Benito Juárez, Cuauhtémoc, Venustiano Carranza y Miguel Hidalgo.

B) El primer contorno. Se considera con base en el crecimiento que la ciudad experimentó entre 1930 a 1950. Formado a partir de las delegaciones: Azcapotzalco, Coyoacán, Cuajimalpa, Gustavo A. Madero, Iztacalco, Iztapalapa y Álvaro Obregón.

C) El segundo contorno. Coincide, aproximadamente, con el crecimiento urbano dado entre 1950 y 1970, en el cual se incorporan cuatro delegaciones más: La Magdalena Contreras, Tlahúac, Tlalpan y Xochimilco.

D) El tercer contorno. Formado a partir de 1970, se une la delegación Milpa Alta. 
Así para 1990 las delegaciones que se observan en los extremos, muy baja pobreza y muy alta pobreza, son Benito Juárez y Milpa Alta (véase cuadro 1). En el caso de las delegaciones con nivel de muy baja pobreza se encuentran la delegación Benito Juárez y Cuauhtémoc, ambas delegaciones forman parte de la ciudad central del DF.

Por otra parte, las delegaciones con los niveles más altos de pobreza o muy alta pobreza corresponden con cinco delegaciones; que para 1990 aún conservaban rasgos tanto en lo económico como en lo político y lo social característicos de las poblaciones rurales. Estas van desde el primer contorno: Cuajimalpa, segundo contorno: La Magdalena Contreras, Xochimilco y Tláhuac y el tercer contorno: Milpa Alta, las cinco delegaciones se localizan en el sur de la ciudad, lo cual nos indica que en 1990 conforme nos acercamos al sur del DF la probabilidad de encontrar delegaciones con rasgos de pobreza es mayor. Cabe señalar que según el orden de nivel de pobreza alcanzado por las delegaciones, Benito Juárez es la delegación con menor proporción de pobreza y Milpa Alta, por el contrario, es la que cuenta con el mayor nivel de pobreza en el DF.

\section{Plano 1}

\section{Ubicación geográfica de las delegaciones del DF}

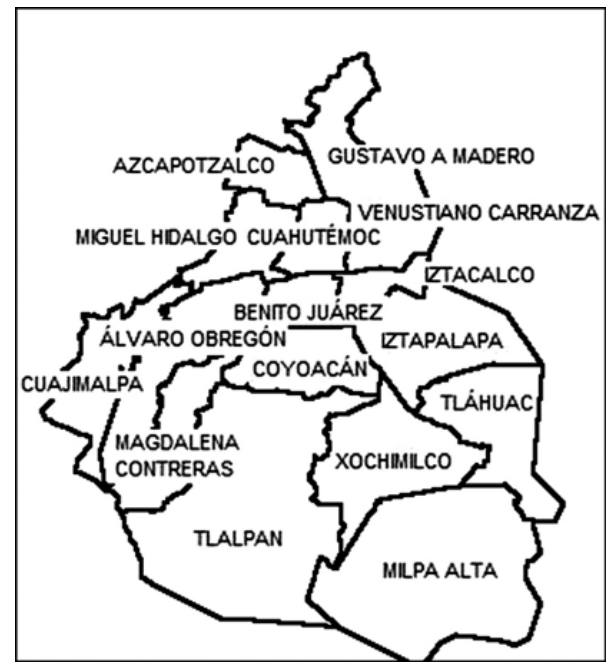

Fuente: Elaboración propia. 
Polis, Revista de la Universidad Bolivariana, Volumen 10, $N^{\circ}$ 29, 2011

\section{Cuadro 1 \\ Orden y nivel espacial de pobreza por delegaciones en el DF 1990}

\begin{tabular}{|c|c|c|c|c|c|}
\hline DELEGACIÓN & $\begin{array}{l}\text { MUY } \\
\text { BAJA }\end{array}$ & BAJA & MEDIA & ALTA & $\begin{array}{l}\text { MUY } \\
\text { ALTA }\end{array}$ \\
\hline BENITO JUAREZ & 1 & & & & \\
\hline COYOACAN & & 4 & & & \\
\hline CUAUHTEMOC & & 2 & & & \\
\hline MIGUELLHIDALGO & & 3 & & & \\
\hline AZCAPOTZALCO & & & 6 & & \\
\hline $\begin{array}{l}\text { VENUSTIANO } \\
\text { CARRANZA }\end{array}$ & & 5 & & & \\
\hline IZTACALCO & & & 8 & & \\
\hline GUSTAVO A. MADERO & & & 7 & & \\
\hline ALVARO OBREGON & & & 10 & & \\
\hline TLALPAN & & & 11 & & \\
\hline IZTAPALAPA & & & 9 & & \\
\hline $\begin{array}{l}\text { CUAJIMALPADE } \\
\text { MORELOS }\end{array}$ & & & & 14 & \\
\hline TLAHUAC & & & & 15 & \\
\hline $\begin{array}{l}\text { MAGDALENA } \\
\text { CONTRERAS }\end{array}$ & & & & 12 & \\
\hline XOCHIMILCO & & & & 13 & \\
\hline MILPAALTA & & & & & 16 \\
\hline
\end{tabular}

Fuente: elaboración propia con base en (INEGI, 1990)

En el transcurso entre 1990 y el 2000 se aplicaron, principalmente entre 1998-2000, programas y medidas de política social encaminadas a la disminución de la pobreza. Tales medidas, la llegada y salida de habitantes del DF y los cambios en los niveles educativos y condiciones de empleo de mujeres y hombres, permearon el cambio experimentado en los niveles de pobreza existentes según unidades geográficas.

Tal proceso condujo a una cierta clase de homogeneización del espacio, en el sentido de que la mayor parte de las delegaciones -14 de las 16- se concentran en los niveles de baja, media y alta pobreza (véase cuadro 2), reduciendo el número de delegaciones que se ubicaban en los extremos, lo cual da muestra de que en algunas delegaciones se redujeron los niveles de pobreza pero en otras estos aumentaron. Así, la única delegación que reportó muy baja pobreza tanto en 1990 como en el 2000 es la Benito Juárez ubicada en la ciudad central, asimismo, la delegación que mantuvo una situación desfavorable -muy baja pobreza- entre 1990-2000 corresponde con Milpa Alta ubicada en el tercer contorno. 
Si bien, tanto para 1990 como para el 2000, las delegaciones con situación de pobreza significativa eran, excepto las delegaciones de la ciudad central, Gustavo A Madero, Iztacalco, Azcapotzalco, Iztapalapa, Álvaro Obregón, Tlalpan, La Magdalena Contreras, Xochimilco, Cuajimalpa y Tláhuac, la distribución de la misma cambio para el 2000: la delegación Venustiano Carranza aumento en sus niveles de pobreza en grado tal que pasó de baja pobreza a un nivel de media pobreza y por debajo de la delegación Azcapotzalco.

\section{Cuadro 2 \\ Orden y nivel espacial de pobreza por delegaciones en el DF 2000}

\begin{tabular}{|l|r|r|r|r|r|}
\hline DELEGACIÓN & MUY BAJA & BAJA & MEDIA & ALTA & MUY ALTA \\
\hline BENITO JUAREZ & 1 & & & & \\
\hline COYOACAN & & 2 & & & \\
\hline CUAUHTEMOC & & 3 & & & \\
\hline MIGUEL HIDALGO & & 4 & & & \\
\hline AZCAPOTZALCO & & & 5 & & \\
\hline VENUSTIANO CARRANZA & & & 6 & & \\
\hline IZTACALCO & & & 7 & & \\
\hline GUSTAVO A. MADERO & & & 8 & & \\
\hline ALVARO OBREGON & & & 9 & & \\
\hline TLALPAN & & & 10 & & \\
\hline IZTAPALAPA & & & & 11 & \\
\hline CUAJMALPA DE MORELOS & & & & 12 & \\
\hline TLAHUAC & & & & 13 & \\
\hline MAGDALENA CONTRERAS & & & & 14 & \\
\hline XOCHIMLCO & & & & 15 & \\
\hline MILPA ALTA & & & & & \\
\hline \hline
\end{tabular}

Fuente: elaboración propia con base en (INEGI, 2000).

A su vez, se observa que ciertas delegaciones se mantuvieron en un mismo nivel de pobreza entre 1990-2000, sin embargo, se registro un avance o tendencia a la reducción de tales niveles, como es el caso de la delegación Álvaro Obregón, la cual paso del sitio 10 en 1990 al 9 en 2000. Por el contrario, existen unidades geográficas como la delegación Iztapalapa donde aumento la pobreza al pasar del sitio 9 en 1990 al 11 en el 2000 (véase grafica 1$)$. 


\section{Gráfica 1}

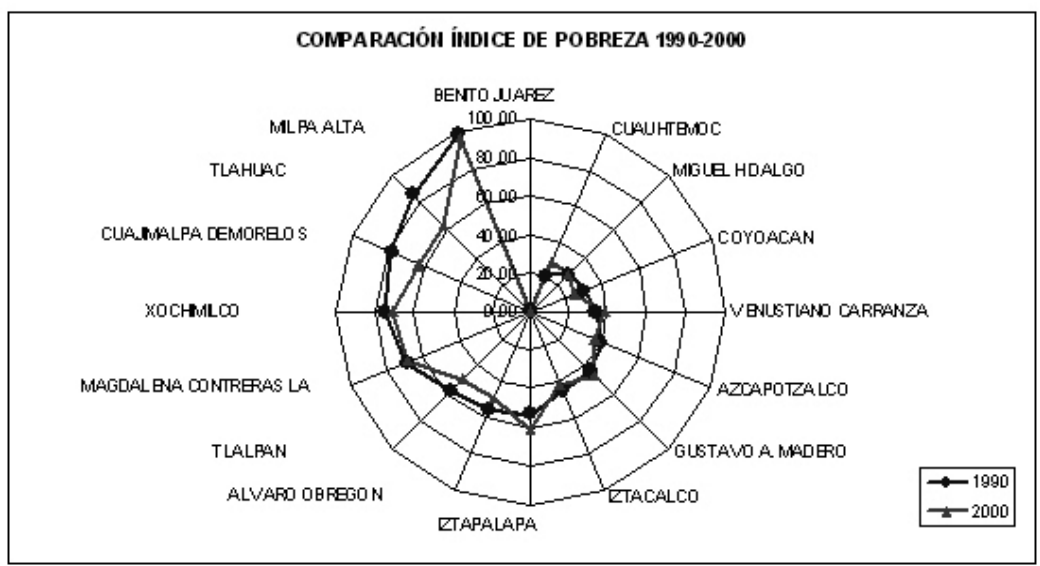

Fuente: Elaboración propia.

Los cambios de mayor proporción en la reducción de la pobreza corresponden con las delegaciones: Álvaro Obregón y Cuajimalpa, ubicadas en el primer contorno, Tlalpan y Tláhuac localizadas en el segundo contorno. Y los cambios más significativos que implican un aumento en el nivel de pobreza; corresponden con las delegaciones Cuahutémoc, Venustiano Carranza e Iztapalapa, las dos primeras forman parte de la ciudad central y la segunda del primer contorno.

Esto conduce a argumentar que en 1990 mientras se transita de la ciudad central al tercer contorno en esa misma dimensión aumenta la proporción de delegaciones que cuentan con niveles altos de pobreza. Porque son las delegaciones que forman ciudad central las que cuentan con los niveles de menor cuantía de pobreza, y son las delegaciones del segundo contorno y Milpa Alta las delegaciones que cuentan con niveles de pobreza altamente significativos.

Para el 2000, si bien existe una serie de transformaciones, tanto en el orden como en los niveles de pobreza, en algunas delegaciones, se puede observar que la distribución de la pobreza según el nivel de ordenamiento por contornos se mantiene, es decir, la probabilidad de ubicar delegaciones altamente pobres en la ciudad central y el primer contorno es baja: Cuajimalpa es la excepción. No así para las delegaciones que se localizan entre el segundo y tercer contornos las cuales cuentan con niveles alto o muy alto de pobreza. Por lo tanto, para 1990-2000 es plausible argumentar que conforme se pasa de la ciudad central al tercer contorno los niveles de pobreza aumentan en el DF, esto refleja en mucho el hecho de que las delegaciones con mayores niveles de pobreza se localican al sur de la ciudad y 
las que cuentan con niveles de pobreza menores correspondan con el centro y norte del DF.

Observando los cambios en su conjunto, se tiene que en el caso de las delegaciones que en 1990 se encontraban en el nivel de muy baja pobreza; sólo Benito Juárez se mantuvo en un nivel de muy baja pobreza, aunado a una disminución en su nivel de pobreza imperante. Esto, se debio principalmente al aumento en el nivel eduactivo de los hombres y mujeres residentes en tal delegación y su incorporación a empleos mejor remunerados (véase cuadro 3). Por su parte, la delegación Cuahutémoc experimento un retroceso al incrementerse su nivel de pobreza y con ello, pasó de un nivel de muy baja pobreza en 1990 a un nivel de baja pobreza en el 2000.

En el caso de las delegaciones que reportaban altos niveles de pobreza en 1990 se observa que para el 2000, Xochimilco, Cuajimalpa y Tláhuac existe una disminución en el nivel de pobreza (véase cuadro 3). Sin embargo, en el caso de Milpa Alta se observa una reducción en el nivel de pobreza que no reduitua en su transformación en delegación con un nivel de muy alta pobreza, así Milpa Alta era en 1990 y es en el 2000 la única delegación con un nivel de muy alta pobreza en comparación con el resto de las delegaciones del DF. El resto de delegaciones experimentaron aumentos o disminuciones en su proporción de pobreza. En el primer caso se tiene a las delegaciones: Miguel Hidalgo, Venustiano Carranza, Gustavo A. Madero e Iztapalapa. Y en el segundo caso se registran las delegaciones: Coyoacán, Azcapotzalco, Iztacalco, Álvaro Obregón, Tlalpan y La Magdalena Contreras.

\section{Cuadro 3}

\section{Cambio en el nivel de pobreza entre 1990 y 2000}

\begin{tabular}{|l|c|}
\hline DELEGACIÓN & CAMBIO \\
\hline BENITO JUAREZ & DISMINUCIÓN \\
\hline CUAUHTEMOC & AUMENTO \\
\hline MIGUEL HIDALGO & AUMENTO \\
\hline COYOACAN & DISMINUCIÓN \\
\hline VENUSTIANO CARRANZA & AUMENTO \\
\hline AZCAPOTZALCO & DISMINUCIÓN \\
\hline GUSTAVO A. MADERO & AUMENTO \\
\hline ZTTACALCO & DISMINUCIÓN \\
\hline IZTAPALAPA & AUMENTO \\
\hline ALVARO OBREGON & DISMINUCIÓN \\
\hline TLALPAN & DISMINUCIÓN \\
\hline LA MAGDALENA CONTRERAS & DISMINUCIÓN \\
\hline XOCHIMILCO & DISMINUCIÓN \\
\hline CUAJIMALPA DE MORELOS & DISMINUCIÓN \\
\hline TLAHUAC & DISMINUCIÓN \\
\hline MILPA ALTA & DISMINUCIÓN \\
\hline
\end{tabular}

Fuente: Elaboación propia. 


\section{Zonas Espaciales de Pobreza 1990-2040}

Una vez identificado el nivel de pobreza y la existencia de una reducción o aumento de la misma, se plantea la plausibilidad de identificar zonas espaciales de pobreza que conduzcan a la integración de un sistema territorial de continuo que permita observar la dinámica espacial de la pobreza en el DF entre 1990-2000 y un pronóstico que va del 2010 al 2040 que induzca a estipular la probabilidad de convergencia del sistema en su conjunto o de algunas delegaciones aledañas.

En este sentido, se forman Zonas Espaciales de Pobreza (ZEP), las cuales se basan en los niveles de pobreza existentes en cada una de las delegaciones. Así, para 1990 (véase plano 2) se identifican cuatro ZEP: La primera ZEP, en 1990, corresponde con los niveles de muy baja y baja pobreza que contempla las delegaciones centrales: Benito Juárez, Miguel Hidalgo, Cuauhtémoc y Venustiano Carranza con la suma de la delegación Coyoacán. La segunda ZEP comprende delegaciones con alto y muy alto nivel de pobreza: Milpa Alta, Tláhuac y Xochimilco, localizadas al sur oriente del DF. Aunado a ellas, sólo pueden integrarse dos ZEP más, estas se forman a partir de la agregación de dos delegaciones aledañas: la ZEP formada por Iztapalapa e Iztacalco y la integrada por las delegaciones Gustavo A Madero y Azcapotzalco. Las otras cuatro delegaciones localizadas al sur poniente y sur del DF no se pueden integrar de acuerdo con los niveles de pobreza que sustentan: Cuajimalpa, La Magdalena Contreras, Tlalpan y Álvaro Obregón.

\section{Plano 2 \\ Distribución espacial de la pobreza en el DF 1990}

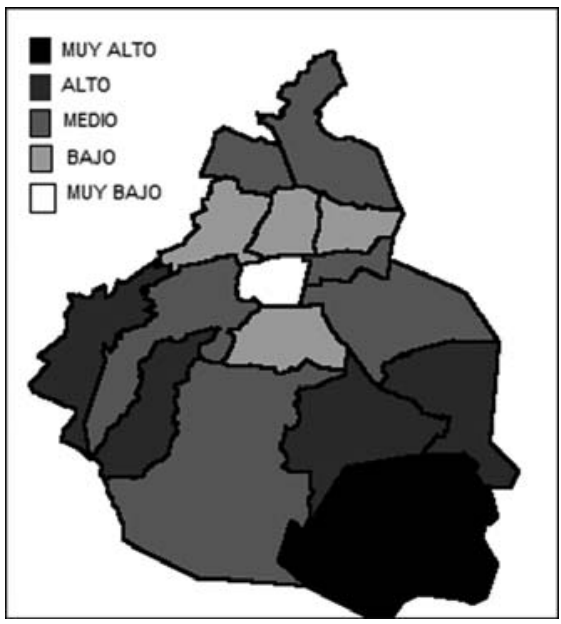

Fuente: Elaboración propia con base en cuadro 1. 
Cabe señalar que sólo pueden ser integradas cuatro ZEP, una de muy baja y baja pobreza, dos más de nivel medio de pobreza y la restante que comprende niveles de pobreza de muy alto y alto.

Para el 2000 se observan sólo tres ZEP (véase plano 3), debido principalmente a la integración de las delegaciones Iztacalco e Iztapalapa a otras ZEP. Estas corresponden con la ZEP de muy bajo y bajo nivel de pobreza que es similar que la ZEP en 1990 a excepción de que la delegación Venustiano Carranza al incrementar sus niveles de pobreza deja de pertenecer a esta ZEP. La segunda, que comprende los niveles de muy alta y alta pobreza se extiende al sumarse la delegación Iztapalapa, conformando la ZEP: Milpa Alta, Xochimilco, Tláhuac e Iztapalapa y localizada al sur y centro oriente del DF. Esta ZEP tiene que ser tomada en cuenta en mayor medida por los planificadores que estudian, a través de acciones concretas, la reducción de la pobreza en el DF, puesto que Iztapalapa aumento sus niveles de pobreza y ello contribuye a que sea considerada en esta ZEP de peores condiciones de vida.

La ZEP restante, corresponde con las delegaciones que se ubican al norte del DF, puesto que debido al aumento de la pobreza en la delegación Venustiano Carranza se permitió la incorporación de la delegación Iztacalco al par Gustavo A Madero-Azcapotzalco. Esto implica que conforme pasa el tiempo el territorio se integra y homogeniza en mayor cuantía, pues ahora se tiene un territorio más integrado y con un menor número de ZEP. Sin embargo, las delegaciones no integradas en 1990 siguen siendo las mismas en el 2000.

\section{Plano 3 \\ Distribución espacial de la pobreza en el DF 2000}

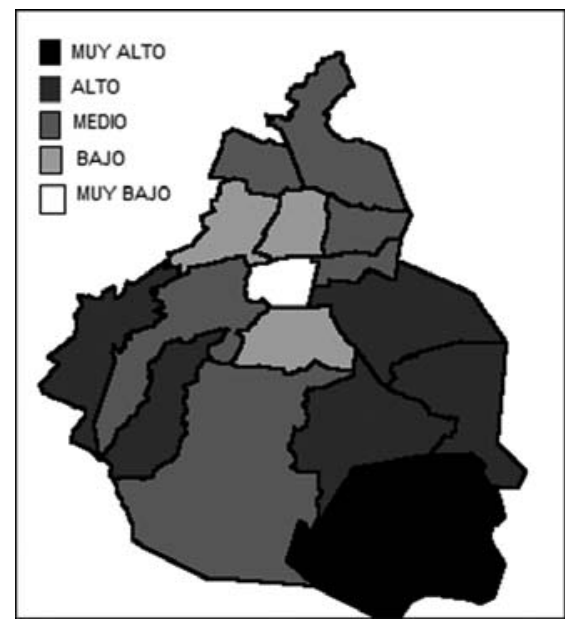

Fuente: Elaboración propia con base en cuadro 2. 
Ahora, la disminución de la pobreza en varias delegaciones principalmente se debió al incremento en los niveles educativos, esto es mayor alfabetización y mayor asistencia a la escuela por parte de los menores. Esto por supuesto puede impactar favorablemente en un futuro en los niveles de pobreza de las familias en estado de precariedad, al aumentar sus ingresos y con ello, existe la alta probabilidad de que dejen de ser pobres.

Estos mayores ingresos redundan en la construcción o ampliación o reparación de sus viviendas, pudiendo utilizar los créditos que el Gobierno del DF otorga, pues en este caso ya cuentan con los recursos monetarios para cumplir con compromisos de este tipo. Empero, los aumentos en los niveles de pobreza, que corresponden con el 31.2\% de las delegaciones, se deben principalmente al deterioro del indicador de feminización de la pobreza empleado, es decir, con la merma en las capacidades de las mujeres que residen en estas delegaciones para la satisfacción de sus necesidades básicas.

Ahora lo que resta es establecer un pronóstico hasta el 2040 de lo que ocurrirá en el DF en materia de pobreza (véase cuadro 4). Para ello, veamos primero las distancias porcentuales estimadas para las delegaciones, en donde, la distancia máxima es la que se identifica entre la delegación con menor nivel de pobreza que es la delegación Benito Juárez y la delegación con mayor nivel de pobreza que es la delegación Milpa Alta -en este caso se fija a Milpa Alta y lo que se observa es que tanto se acercan o alejan los valores porcentuales de las delegaciones respecto de la delegación Milpa Alta.

En primer término se tienen las delegaciones en donde desde 1990 hasta el 2040 verán aumentada su distancia de pobreza, lo que indica es que tienden a una erradicación de la pobreza: Tlalpan, Álvaro Obregón, Xochimilco, Tláhuac y Cuajimalpa. Por el otro lado, se tienen las delegaciones que experimentan de 1990 al 2000 una disminución en sus distancias respecto de Milpa Alta y posteriormente aumentan hasta el 2040, estas delegaciones son: Benito Juárez, Miguel Hidalgo, Venustiano Carranza, Azcapotzalco, Gustavo A Madero, Iztacalco, Coyoacán, La Magdalena Contreras, Iztapalapa y Cuauhtémoc. De hecho el aumento de la distancia respecto de Milpa Alta es un claro signo de que las cosas mejorarán para todas las delegaciones algunas con mayores aumentos que otras. Esto bajo el contexto de que se aplique una política débil en el combate a la pobreza por parte del Gobierno del DF. Es decir, aunque los esfuerzos no sean sustanciales el nivel de pobreza descenderá, pero su erradicación queda cuestionada. 


\section{Cuadro 4 \\ Distancias porcentuales de una política débil de combate a la pobreza en las delegaciones del DF 1990-2040}

\begin{tabular}{|l|r|r|r|r|r|r|}
\hline DELEGACION & 1990 & 2000 & 2010 & 2020 & 2030 & 2040 \\
\hline BENITO JUAREZ & 3.63 & 1.08 & 1.03 & 1.02 & 0.97 & 0.68 \\
\hline CUAUHTEMOC & 5.85 & 9.53 & 9.23 & 9.20 & 9.09 & 8.66 \\
\hline MIGUEL HIDALGO & 7.51 & 9.44 & 9.15 & 9.12 & 9.01 & 8.58 \\
\hline COYOACAN & 7.31 & 7.52 & 7.28 & 7.25 & 7.17 & 6.83 \\
\hline $\begin{array}{l}\text { VENUSTIANO } \\
\text { CARRANZA }\end{array}$ & 11.11 & 16.81 & 16.36 & 16.28 & 16.09 & 15.31 \\
\hline AZCAPOTZALCO & 14.11 & 15.20 & 14.78 & 14.72 & 14.54 & 13.85 \\
\hline $\begin{array}{l}\text { GUSTAVO A. } \\
\text { MADERO }\end{array}$ & 17.00 & 24.01 & 23.44 & 23.32 & 23.03 & 21.91 \\
\hline IZTACALCO & 18.01 & 18.51 & 18.03 & 17.94 & 17.72 & 16.87 \\
\hline IZTAPALAPA & 26.26 & 39.36 & 38.59 & 38.35 & 37.84 & 35.98 \\
\hline ALVARO OBREGON & 27.80 & 26.07 & 25.46 & 25.33 & 25.01 & 23.79 \\
\hline TLALPAN & 32.45 & 26.32 & 25.71 & 25.58 & 25.25 & 24.02 \\
\hline $\begin{array}{l}\text { MAGDALENA } \\
\text { CONTRERAS }\end{array}$ & 45.53 & 49.87 & 49.04 & 48.71 & 48.03 & 45.65 \\
\hline XOCHIMILCO & 56.11 & 54.53 & 53.69 & 53.33 & 52.57 & 49.96 \\
\hline $\begin{array}{l}\text { CUAJMALPA DE } \\
\text { MORELOS }\end{array}$ & 60.29 & 42.04 & 41.25 & 40.99 & 40.44 & 38.45 \\
\hline TLAHUAC & 76.28 & 42.72 & 41.92 & 41.66 & 41.09 & 39.07 \\
\hline MILPA ALTA & 100.00 & 100.00 & 100.00 & 100.00 & 100.00 & 100.00 \\
\hline
\end{tabular}

Fuente: elaboración propia.

Ahora para observar la diferencia entre la aplicación de una política débil o fuerte en el combate a la pobreza se muestran las siguientes dos gráficas (véanse gráfica 2 y 3). En la primera de ellas corresponde con la aplicación de una política débil hasta el 2010, lo cual sólo trae consigo una somera reducción en el nivel de pobreza en las delegaciones del DF. Sin embargo, si se aplicará una política fuerte de combate a la pobreza los resultados de disminución de la pobreza son sustanciales. Porque mientras una política débil no erradica la pobreza una política fuerte si lo hace y con ello mejora los niveles de vida de los pobladores de estas delegaciones. 
Polis, Revista de la Universidad Bolivariana, Volumen 10, $N^{\circ}$ 29, 2011

\section{Gráfica 2}

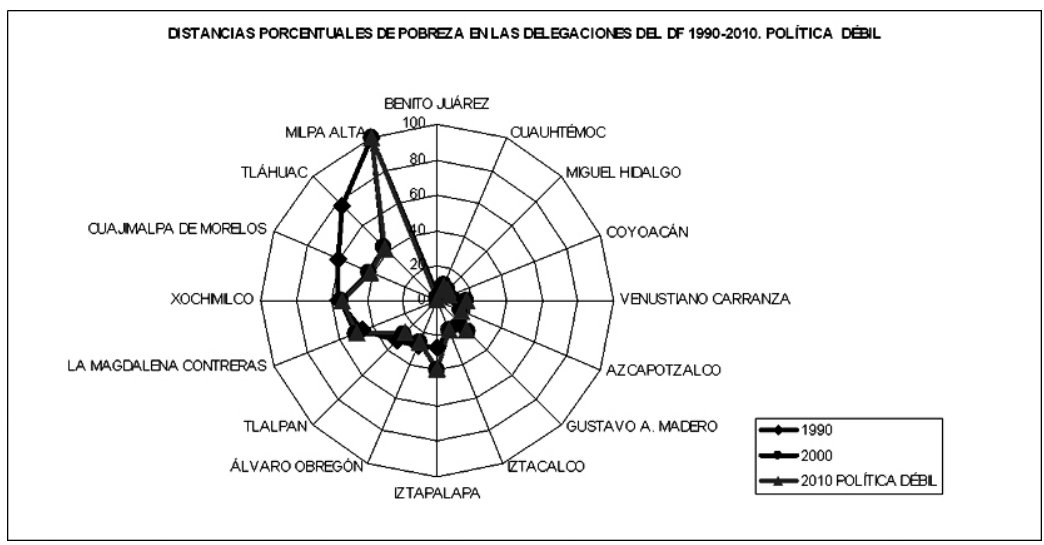

Fuente: elaboración propia.

\section{Gráfica 3}

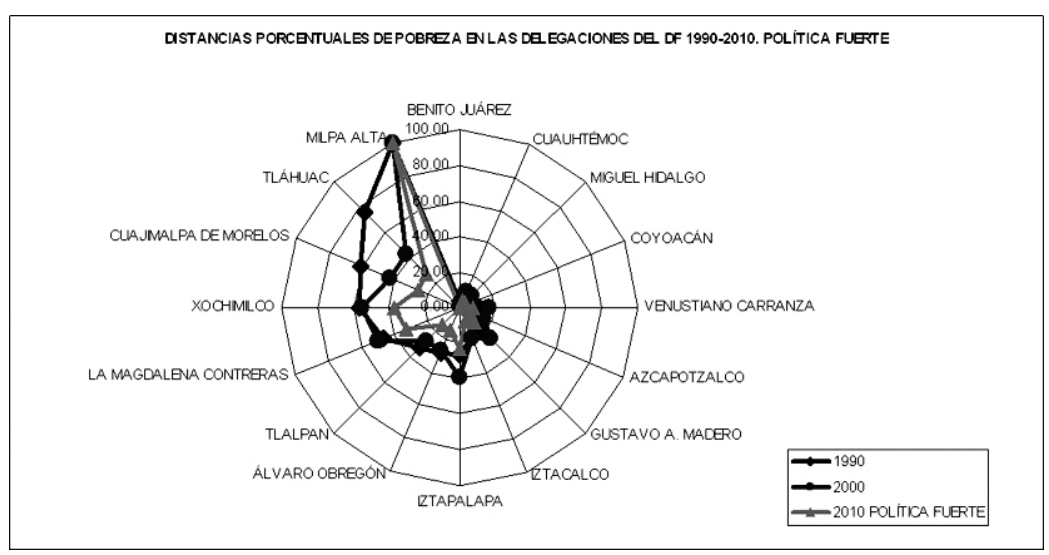

Fuente: elaboración propia.

Estas transformaciones pueden ser visualizadas a través del orden y nivel espacial de las delegaciones y los planos respectivos. Así, en el primer caso tenemos que para el 2010 la mitad de las delegaciones se concentran en el nivel medio de pobreza (véase cuadro 5) y que las delegaciones ubicadas en los extremos de muy baja y muy alta pobreza continúan siendo Benito Juárez y Milpa Alta respectivamente. 


\section{Cuadro 5 \\ Orden y nivel espacial de pobreza por delegaciones en el DF 2010}

\begin{tabular}{|l|r|r|r|r|r|}
\hline DELEGACION & MUY BANA & \multicolumn{1}{|c|}{ BAA } & MEDIA & \multicolumn{1}{c|}{ ALTA } & MUY ALTA \\
\hline BENITO JUAREZ & 1 & & & & \\
\hline COYOACAN & & 2 & & & \\
\hline CUAUHTEMOC & & 3 & & & \\
\hline MIGUEL HIDALGO & & 4 & & & \\
\hline AZCAPOTZALCO & & & 5 & & \\
\hline $\begin{array}{l}\text { VENUSTIANO } \\
\text { CARRANZA }\end{array}$ & & & 6 & & \\
\hline IZTACALCO & & & 7 & & \\
\hline GUSTAVOA. MADERO & & & 8 & & \\
\hline ALVARO OBREGON & & & 9 & & \\
\hline TLALPAN & & & 10 & & \\
\hline IZTAPALAPA & & & 11 & & \\
\hline $\begin{array}{l}\text { CUAJIMALPADE } \\
\text { MORELOS }\end{array}$ & & & 12 & & \\
\hline TLAHUAC & & & & 13 & \\
\hline $\begin{array}{l}\text { MAGDALENA } \\
\text { CONTRERAS }\end{array}$ & & & & 14 & \\
\hline XOCHIMILCO & & & & & \\
\hline MILPAALTA & & & & & \\
\hline
\end{tabular}

Fuente: elaboración propia.

Para el 2020 se pronostica (véase cuadro 6), ceteris paribus, que la mayor concentración de delegaciones corresponderá con el nivel medio -12 de ellas-, y sólo una delegación permanecerá en el nivel de baja pobreza: Coyoacán. Esto implica que las delegaciones Cuahutémoc y Miguel Hidalgo pasaran a formar parte de las delegaciones con nivel medio de pobreza, esto se debe a que si bien sus distancias respecto a Milpa Alta aumentan y respecto a la delegación Benito Juárez disminuyen, la disminución de la pobreza en Benito Juárez es de tal magnitud que "arrastra” las cifras de las delegaciones antes mencionadas hasta colocarlas con un nivel más elevado de pobreza.

El caso para las delegaciones de baja pobreza Tláhuac y La Magdalena Contreras corresponde con lo contrario, se estima que dadas las magnitudes de beneficios vertidos por el Gobierno del DF a estas las colocara en una mejor situación reduciendo sus niveles de pobreza imperantes. Los casos que continúan en un orden jerárquico similar son la de legación Benito Juárez con muy bajo nivel de pobreza y la delegación Milpa Alta con un muy alto nivel de pobreza. De hecho la situación que experimentan las delegaciones permite argumentar sobre la existencia de convergencia en los niveles de pobreza, excluyendo los bajísimos niveles de pobreza de la delegación Benito Juárez. 


\section{Cuadro 6 \\ Orden y nivel espacial de pobreza por delegaciones en el DF 2020}

\begin{tabular}{|c|c|c|c|c|c|}
\hline DELEGACTÓN & $\begin{array}{l}\text { MUY } \\
\text { BAJA }\end{array}$ & BAJA & MEDIA & ALTA & MUY ALTA \\
\hline BENTO JUAREZ & 1 & & & & \\
\hline COYOACAN & & 2 & & & \\
\hline CUAUHTEMOC & & & 3 & & \\
\hline MIGUEL HIDALGO & & & 4 & & \\
\hline AZCAPOTZALCO & & & 5 & & \\
\hline VENUSTIANO CARRANZA & & & 6 & & \\
\hline IZTACALCO & & & 7 & & \\
\hline GUSTAVO A. MADERO & & & 8 & & \\
\hline ALVARO OBREGON & & & 9 & & \\
\hline TLALPAN & & & 10 & & \\
\hline IZTAPALAPA & & & 11 & & \\
\hline CUAIMALPA DE MORELOS & & & 12 & & \\
\hline TLAHUAC & & & 13 & & \\
\hline MAGDALENA CONTRERAS & & & 14 & & \\
\hline XOCHIMLCO & & & & 15 & \\
\hline $\begin{array}{l}\text { MLPA ALTA } \\
\end{array}$ & & & & & 16 \\
\hline
\end{tabular}

Fuente: elaboración propia.

Para el 2030 (véase cuadro 7) las especulaciones sobre convergencia son más realistas, esto indica que conforme pasa el tiempo las distancias entre las delegaciones se acortan, y que a su vez, la delegación Benito Juárez reducirá aún más la pobreza existente allí, esto se debe en gran medida a las mejoras en los niveles educativos y a la mejora en la relación entre los géneros que se espera ocurran en el DF. La delegación Milpa Alta se mantendrá en el nivel de muy alta pobreza. 


\section{Cuadro 7 \\ Orden y nivel espacial de pobreza por delegaciones en el DF 2030}

\begin{tabular}{|c|c|c|c|c|c|}
\hline \multirow{2}{*}{\multicolumn{6}{|c|}{$\begin{array}{l}\text { DELEGACION } \\
\text { BENITO JUAREZ }\end{array}$}} \\
\hline & & & & & \\
\hline \multicolumn{6}{|l|}{ COYOACAN } \\
\hline \multicolumn{6}{|l|}{ CUAUHTEMOC } \\
\hline \multicolumn{6}{|l|}{ MIOUEL HIDALOO } \\
\hline \multicolumn{6}{|l|}{ AZCAPOTZALCO } \\
\hline \multicolumn{6}{|l|}{ VENUSTIANO CARRANZA } \\
\hline \multicolumn{6}{|l|}{ Iztacalco } \\
\hline \multicolumn{6}{|l|}{ GUSTAVO A MADERO } \\
\hline \multicolumn{6}{|l|}{ ALVARO OBREGON } \\
\hline \multicolumn{6}{|l|}{ TLALPAN } \\
\hline \multicolumn{6}{|l|}{ IZTAPALAPA } \\
\hline \multicolumn{6}{|l|}{ CUATMALPADE MORELOS } \\
\hline \multicolumn{6}{|l|}{ TLAHUAC } \\
\hline \multicolumn{6}{|l|}{ MAGDALENACONIRERAS } \\
\hline \multicolumn{6}{|l|}{ XOCHIMILCO } \\
\hline MILPAALTA & & & & & \\
\hline
\end{tabular}

Fuente: elaboración propia.

Las anteriores circunstancias nos hacen pensar en la existencia de convergencia en los niveles de pobreza existentes entre los habitantes del DF. Condición que se ve reforzada al observar que para el 2040 (véase cuadro 8) los habitantes de la delegación Milpa Alta en promedio verán experimentar un descenso considerable en su situación de pobreza, porque esta delegación tendrá un nivel inferior de pobreza: alta pobreza.

De hecho se podría esperar que los niveles de pobreza en la delegación disminuyan aún más hasta alcanzar el parámetro de convergencia con el resto de las delegaciones en comparación con la delegación Benito Juárez, pero que al reordenarlos, excluyendo Benito Juárez, tendrán niveles de pobreza aún más bajos. 


\section{Cuadro 8 \\ Orden y nivel espacial de pobreza por delegaciones en el DF 2040}

\begin{tabular}{|l|l|l|l|l|l|}
\hline DELEGACION & MUYBAJA & BAJA & MEDIA & ALTA & MUY ALTA \\
\hline BENITO JUAREZ & 1 & & & & \\
\hline COYOACAN & & & 2 & & \\
\hline CUAUHTEMOC & & & 3 & & \\
\hline MIGUEL HIDALGO & & & 4 & & \\
\hline AZCAPOTZALCO & & & 5 & & \\
\hline VENUSTIANO CARRANZA & & & 6 & & \\
\hline IZTACALCO & & & 7 & & \\
\hline GUSTAVOA. MADERO & & & 8 & & \\
\hline ALVARO OBREGON & & & 9 & & \\
\hline TLALPAN & & & 10 & & \\
\hline IZTAPALAPA & & & 11 & & \\
\hline CUAJIMALPADEMORELOS & & & 12 & & \\
\hline TLAHUAC & & & 13 & & \\
\hline MAGDALENACONTRERAS & & & 14 & & \\
\hline XOCHIMILCO & & & 15 & & \\
\hline MILPAALTA & & & & 16 & \\
\hline
\end{tabular}

Fuente: elaboración propia.

Ahora si visualizamos esto a través de cómo se reconfiguran las regiones de pobreza es posible observar como la convergencia media para una tendencia en la homogeneización de las condiciones de pobreza en el DF respecto de los parámetros de pobreza de la delegación Benito Juárez. Pero que serán levemente heterogéneos respecto de los niveles entre las delegaciones como puede constatarse en el cuadro 4.

Así, en el 2010 existen cuatro regiones (véase plano 4): 1) Azcapotzalco, Gustavo A Madero, Venustiano Carranza, Iztacalco e Iztapalapa, esta última se espera que ocurra una disminución de la pobreza después del sobresalto del 2000. La región 2 queda conformada por Benito Juárez, Coyoacán, Miguel Hidalgo y Cuahutémoc. La región 3, contempla las mismas delegaciones que formaban una región en 1990: Milpa Alta, Tláhuac y Xochimilco. Ahora se forma una nueva región de dos delegaciones y es; Cuajimalpa y Álvaro Obregón, esta se forma por la reducción de los niveles de pobreza esperados en Cuajimalpa. 


\section{Plano 4 \\ Regiones de pobreza para el 2010}

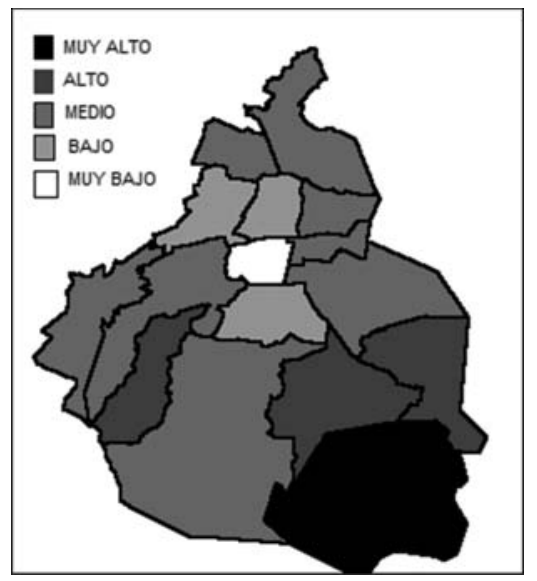

Fuente:

elaboración propia.

Para el 2020 es complejo el argumentar sobre la existencia de regiones debido al proceso de convergencia, en este caso sólo existirían tres regiones, dos propiamente regiones y la última un conglomerado de delegaciones. La primera región esta compuesta por las delegaciones Benito Juárez y Coyoacán y la segunda por las delegaciones Xochimilco y Milpa Alta. El resto de delegaciones como se encuentran dentro de un mismo nivel de pobreza -pero no así de valores de distribución, pues sólo basta ver el cuadro 4 de distancias porcentuales--, forman un conglomerado.

\section{Plano 5 \\ Regiones de pobreza para el 2020}

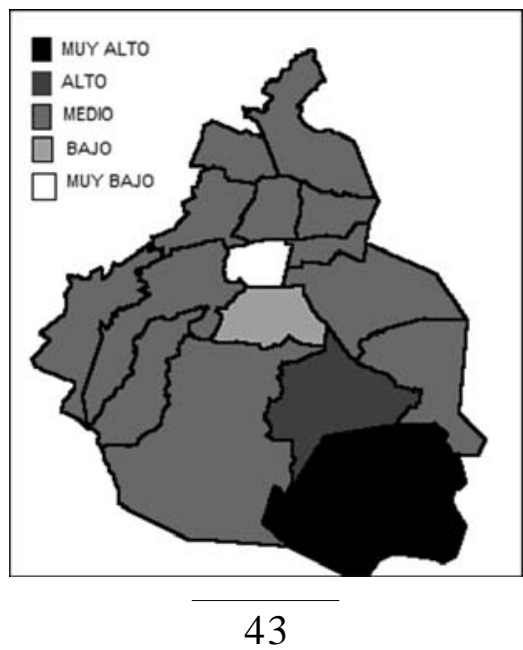

Fuente:

elaboración propia. 
El proceso de la convergencia es tal que para el 2030 y 2040 (véanse planos 6 y 7) ya no se puede hablar de regiones, sino tan sólo de delegaciones. Son dos las delegaciones en los extremos: Benito Juárez y Milpa Alta, aunque en el 2040 los habitantes de la delegación Milpa Alta tienen menores niveles de pobreza.

Claro es que si tomamos consideramos la no inclusión de la delegación Benito Juárez en la métrica los valores se redistribuirán, puesto que como lo indica el cuadro 4 de distancias porcentuales, tanto para el 2030 como para el 2040 los valores asociados serán diferentes. Sin embargo, manteniendo en el sistema los valores de la delegación Benito Juárez el resultado es el observado, pareciese que las delegaciones se agrupan en un mismo nivel porque tienen similares valores asociados, lo cual no es verdad -como ya se mencionó arriba.

\section{Plano 6 \\ Regiones de pobreza para el 2030}

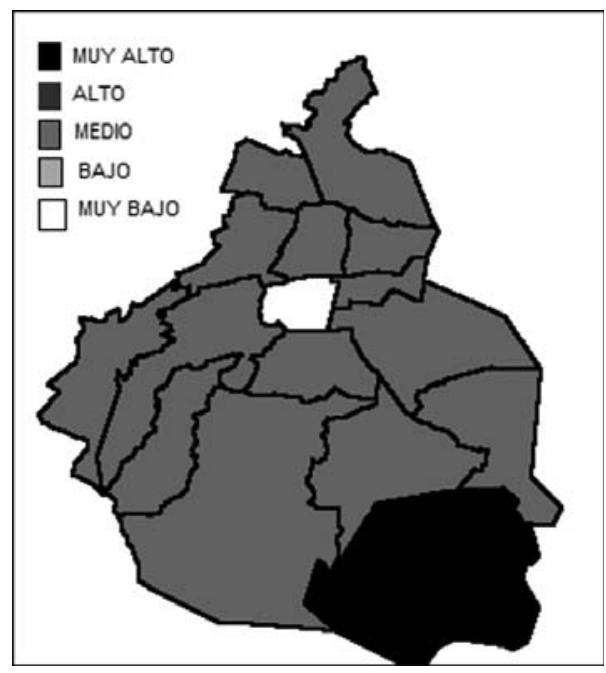

Fuente: elaboración propia. 


\section{Plano 7 \\ Regiones de pobreza para el 2040}

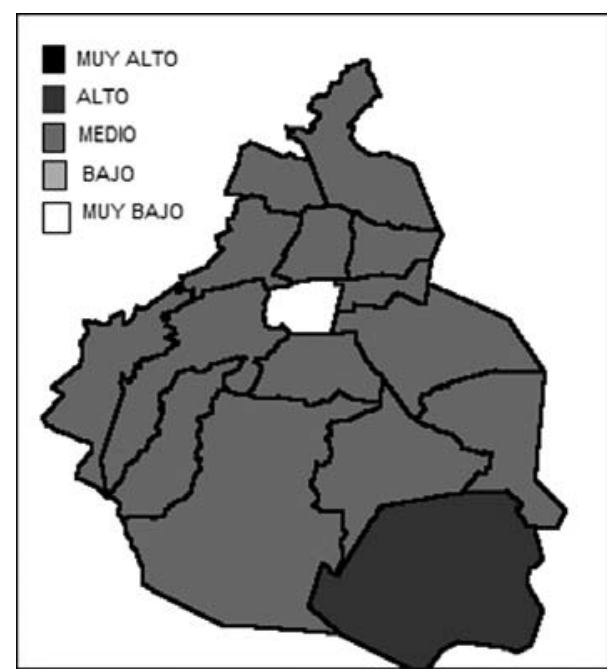

Fuente: elaboración propia.

Lo anterior, muestra como para la determinación de los niveles de pobreza no sólo basta con las agrupaciones hechas para las delegaciones, sino que existen diferencias que se mantienen hasta el 2040, y que sólo pueden ser vistas a través de las distancias porcentuales.

De cualquier manera, las distancias porcentuales y la dinámica de regiones de pobreza indican que tales niveles se van a reducir en el DF, y que de aplicarse una política débil de combate a la pobreza, los resultados de reducción de la pobreza serán mínimos y esta se prolongara por más tiempo.

Ahora si bien se esperan reducciones en los niveles de pobreza, no deja de estar presente una cuestión: aún en el 2040 no se erradicara del todo la pobreza en el DF a menos que la política de combate a la pobreza sea fuerte, es decir, se ataquen los principales problemas que se tienen en educación y vivienda. De hecho los mayores esfuerzos tienen que estar encaminados a mejora en las condiciones de las viviendas: materiales y de servicios y en la elevación de los niveles educativos acompañados de la generación de los empleos necesarios para estas nuevas generaciones, porque generar capital humano y no generar los empleos es cuestión ociosa.

\section{Conclusiones}

La pobreza es una condición que merma la capacidad de los individuos para satisfacer sus necesidades básicas, en este sentido, se observa 
que en algunas delegaciones entre 1990-2000 disminuyó la pobreza, sin embargo, en otras, como es el caso de la delegación Cuauhtémoc, aumenta.

La propia dinámica de la pobreza como referente de las características de los asentamientos humanos refleja que tanto en 1990 como en el 2000 mientras una delegación corresponda con la ciudad central o sea del primer contorno localizada en el norte de la ciudad el nivel de pobreza que reporta es de bajo o muy bajo. Por su parte, para el periodo, si una delegación se localiza en el sur de la ciudad sin importar al contorno que pertenezca; principalmente segundo y tercer contorno, es altamente probable que se encuentre en un nivel de pobreza alto o muy alto.

Tal dinámica de la pobreza refleja que son dos las delegaciones que se localizan en los extremos las cuales se mantienen en su nivel de pobreza entre 1990-2000. La delegación Benito Juárez con un nivel de muy baja pobreza y la delegación Milpa Alta con un reporte de pobreza muy alta.

La identificación de zonas de espacialización de la pobreza (ZEP), es relevante cada vez que es posible reconocer de manera general las áreas geográficas que tienen que atenderse para la minimización o solución de la pobreza. Entre 1990-2000 se observa que la modificación en las ZEP ha redundado en la ampliación de la ZEP norte y la ZEP sur-oriente. La primera se debió a la incorporación de una delegación de la ZEP centro a esta: Venustiano Carranza. La ZEP sur-oriente se amplió por el aumento del nivel de pobreza básicamente de la delegación Iztapalapa.

El caso de una de las delegaciones es relevante: Iztapalapa, pues esta delegación dentro del nivel de alta pobreza aumentó su participación, lo que sin duda de no atenderse en la búsqueda de reducción de la pobreza existente, en el mediano plazo, dejara de ser una delegación de alta pobreza para pasar a formar parte del nivel de muy alta pobreza. Aunque los pronósticos, hasta el 2040, reflejan que los niveles de pobreza en esta delegación disminuirán.

Asimismo, la investigación da muestras de la conformación, a través del tiempo, de las condiciones de pobreza operantes en el DF, de tal suerte, que de crecer los asentamientos humanos en una determinada delegación existe una alta probabilidad de que dichos asentamientos correspondan con el nivel general de pobreza, es decir, sí encuentran residencia en la delegación Benito Juárez es muy probable que no sean pobres pero si lo hacen en Milpa Alta es altamente probable que sean pobres, en todo caso las ZEP reflejan la segregación socio espacial general existente entre las delegaciones del DF. 


\section{Notas}

${ }^{1}$ Este fundamento es utilizado en México a través de la Encuesta Ingreso Gasto de los Hogares. Donde, la inclusión de un bien en la canasta alimentaría depende del número de casos reportados por hogares que sean significativos ante las pruebas estadísticas aplicadas.

${ }^{2}$ Reconocemos que existen otros enfoques, para ello véase (Sen; 1992) y (Villarespe; 2002), por ejemplo.

${ }^{3}$ Un Robinson Crusoe puede considerar que tiene una privación relativa en términos de los habitantes de Inglaterra, sin embargo, en el momento en que este interactúa con su medio no manifiesta una privación relativa de necesidades, porque, satisface sus necesidades con base en los recursos disponibles donde habita, en este sentido, su privación relativa es sólo un "sentimiento de privación" sobre un medio-social distinto de su medio-individual o hábitat. Sin entrar en el tema de las grandes y pequeñas robinsonadas a que se refiere Marx en el capital.

${ }^{4}$ Véase (Sen; 1992).

${ }^{5}$ El los setenta Amartya Sen escribe On Economic inequality en 1973 y posteriormente en 1997 reedita este texto con el titulo en español La desigualdad económica. Edición ampliada con una anexo fundamental de E. Foster y Amartya Sen. Dice Sen: "En esta edición ampliada con un anexo sustancial (...) Durante el último cuarto de siglo los problemas de la desigualdad se han vuelto más centrales (y también más discutidos) (...) a la vez ha surgido y florecido una bibliografía técnica enorme - y a menudo formidable-en la teoría pura de la medición y la evaluación de la desigualdad económica (...). El Anexo es sobre todo un esfuerzo por examinar y evaluar el estado actual de la bibliografía analítica sobre la medición de la desigualdad y la pobreza” ((Sen A. , 1973;Sen \& Foster, 1997).

${ }^{6}$ Destacan los trabajos de (Downs, 1957; Olson, 1965; Becker, 1976).

${ }^{7}$ Este indicador consiste en una serie de variables directas e indirectas que permiten observar la existencia o no de pobreza de las mujeres para un territorio determinado. Véase (Briseño, 2004). 
Polis, Revista de la Universidad Bolivariana, Volumen 10, $N^{\circ}$ 29, 2011

\section{Bibliografía}

Banco Mundial (1992), Comercio exterior, vol. 42, núm. 4; abril, México.

Becker, G. (1976), The Economic Approach To Human Behavior. University of Chicago Press, Chicago.

Boltvinik, Julio (1997), “Aspectos conceptuales y metodológicos para el estudio de la pobreza”, en: Schteingart, Martha (coord.); Pobreza, condiciones de vida y salud en la ciudad de México, El Colegio de México, México.

Ídem (1992), “El método de medición integrada de la pobreza. Una propuesta para su desarrollo”, en: Comercio exterior, vol. 42, núm. 4; abril, México.

Ídem y Hernández Laos, Enrique (1999), Pobreza y Distribución del Ingreso en México, siglo XXI editores, México.

Briseño, Nohemí y Caloca, Oscar (2004), “La calidad de vida de las madres solteras acreditadas en el Programa de Mejoramiento de Vivienda en Lote Familiar”, en: Massolo, Alejandra (coomp.) Una mirada de género a la Ciudad de México, RNIU y UAM-Azcapotzalco, México, DF.

CEPAL-PNUD (1992), "Procedimientos para medir la pobreza en América Latina con el método de la línea de pobreza”, en: Comercio exterior, vol. 42, núm. 4; abril, México.

Downs, A. (1957), An Economic Theory of Democracy. Harper, New York.

Giddens, Anthony (1995), La constitución de la sociedad, Amorrortu, Buenos Aires.

Ídem (1998), La tercera vía, Taurus, Madrid.

Gujarati, Damodar (2004), Econometría, Mc Graw Hill, México.

Heller, Agnes (1996), Una revisión de la teoría de las necesidades, Paidós, Barcelona.

INEGI (2000), XII Censo General de Población y Vivienda, 2000, México.

Ídem(1990), XI Censo General de Población y Vivienda, 1990, México.

Lewis, Oscar (1972), Antropología de la pobreza, FCE, México.

Olson, M. (1965), The logic of Collective Action. Public Goods and Theory of Groups. Harvard University Press, Cambridge. 
SEDESOL (1995), Programa para Superar la Pobreza 1995-2000, México.

Sen, Amartya (2000), Desarrollo y libertad, Planeta, México.

Ídem (2000b), Commodities and capabilities, Oxford India Paperbacks, New Delhi.

Ídem (1998), "Progreso y déficit social: algunas cuestiones metodológicas”, en: Desai, Meghnad, Amartya Sen y Julio Boltvinik, Índice de progreso social: una propuesta, México: UNAM.

Ídem (1997), Bienestar, justicia y mercado, Paidós, Barcelona.

Ídem (1995), Nuevo examen de la desigualdad, Alianza, Madrid.

Ídem (1992), “Sobre conceptos y medidas de pobreza”, en: Comercio Exterior, Banco de Comercio Exterior, vol. 42, núm. 4; abril, México.

Ídem (1976), Elección colectiva y bienestar social, Alianza, Madrid.

Ídem (1973), On Economic inequality. Charenton Press, Oxford.

Sen, A., \& Foster, J. E. (1997), La desigualdad económica. Edición ampliada con una anexo fundamental de E. Foster y Amartya Sen. Alianza, Madrid.

Touraine, Alain (1994), Crítica de la Modernidad, FCE, Buenos Aires.

Villarespe, Verónica (2002), Pobreza, Teoría e Historia, Casa Juan Pablos, México.

Recibido: 25.05.2011

Aceptado: 06.07.2011 


\section{ANEXO 1}

El conjunto vivienda y educación para el Método de Componentes Principales (MCP)

El conjunto de vivienda se estructura a partir de los siguientes subconjuntos: a) condiciones de la vivienda, b) servicios en la vivienda y c) relación espacio-integrantes de la vivienda:

Condiciones de la vivienda. Para la construcción de este rubro se consideran tres categorías. A1) Pisos; A2) Muros; A3) Techos.

Servicios en la vivienda. En este caso se consideran los siguientes elementos: B1) Agua entubada; B2) Drenaje; B3) Electricidad.

Relación espacio-integrantes de la vivienda. Este subconjunto corresponde con: C1) Hacinamiento.

En el caso del nivel educativo, este representa la capacidad de acceso a recursos monetarios y no monetarios que permiten la satisfacción de necesidades a través de la adquisición de satisfactores, de donde, se desprende la idea de que a mayor educación menor probabilidad de ser pobre.

Así, el conjunto de educación corresponde con dos subconjuntos: a) La condición de alfabetismo y b) La condición de asistencia escolar.

La condición de alfabetismo. Se relaciona con la población que debe saber leer y escribir o en su caso que no cuenta con esta capacidad. adelante.

D1) Para ello se exige alfabetismo a partir de los nueve años en

La condición de asistencia escolar. Es la población que no asiste pero tiene que asistir a la escuela.

E1) Se exige asistencia escolar de 6 a 14 años y de 15 y más años no debe necesariamente de asistir a la escuela.

El rubro sobre feminización de la pobreza en el DF (Briseño; 2004) comprende una serie de variables que se relacionan con la probabilidad de existencia de mujeres pobres en dichas unidades geográficas.

Con el conjunto de indicadores se procede, por medio del Método de Componentes Principales, a la estimación de los parámetros de pobreza para cada una de las delegaciones del DF. 


\section{ANEXO 2}

\section{Método de Componentes Principales (MCP)}

El objetivo de este método es transformar un espacio de representación $\mathrm{P}$ en un nuevo espacio P', en el cual los datos estén incorrelados, llamados componentes principales. Estas nuevas variables son combinaciones lineales de las variables originales y se derivan en orden decreciente de importancia, de manera que la primera componente principal explique tanta variación en los datos originales como sea posible.

La técnica para encontrar esta transformación es llamada análisis de componentes principales. Es una técnica dirigida por las variables que resulta adecuada cuando las variables surgen sobre un fundamento igual; como es el caso de nuestras variables empleadas en el estudio.

Las nuevas variables componentes principales deben ser tales que: a) No estén correlacionadas, b) La primera componente principal explique tanto de la variabilidad en los datos como sea posible y c) Cada componente subsiguiente tome en cuenta tanto de la variabilidad restante como sea posible. En cuyo caso supongamos que $\mathbf{X}^{\mathrm{T}}=\left[\mathrm{X}_{1}, \ldots, \mathrm{X}_{\mathrm{p}}\right]$ es una variable aleatoria p-dimensional con media $\mu$ y matriz de covarianzas $\Sigma$. El problema es encontrar un nuevo conjunto de variables, sea $Y_{1}, Y_{2}, \ldots, Y_{p}$, las cuales son no correlacionadas y cuyas varianzas son decrecientes de la primera a la última. Cada Yj será una combinación lineal de las X, de manera que:

$$
Y_{j}=a_{1 j} X_{1}+a_{2 j} X_{2}+\ldots+a_{p j} X_{p}=\mathbf{a}_{j}^{T} \mathbf{X}
$$

Donde $\mathbf{a}_{j}^{T}=\left[a_{1 j, \ldots,} a_{p j}\right]$ es un vector de constantes. En este sentido, la ecuación (1) contiene un factor de escala arbitrario, por ende, es plausible imponer una condición de normalización, tal que $\mathbf{a}_{j}^{T} \mathbf{a}_{j}=\sum_{k=1}^{p} a_{k j}^{2}=1$. Esta condición asegura que las distancias en el p-espacio se preservan.

De esta manera, el primer componente principal, $Y_{1}$, se encuentra eligiendo $a_{1}$ de manera tal que la varianza de $Y_{1}$ se maximiza. Es decir, se elige $a_{1}$ de manera tal que se maximice la varianza de $\mathbf{a}_{1}^{T} \mathbf{X}$ sujeta a la condición de normalización $\mathbf{a}_{1}^{T} \mathbf{a}_{1}=1$. Así, el valor máximo de la varianza de $\mathbf{a}_{1}^{T}$ Xentre todos los vectores $\mathbf{a}_{1}$ que satisfacen $\mathbf{a}_{1}^{T} \mathbf{a}_{1}=1$ es igual a $\lambda$, el eigenvalor más grande de $\Sigma$, esto ocurre cuando $\mathbf{a}_{1}$ es un eigenvector de $\Sigma$ correspondiente al eigenvalor $\lambda_{1}$.

La segunda componente principal, $\mathrm{Y}^{2}$, se encuentra eligiendo $\mathbf{a}_{2}$ de manera tal que Y2 tenga la mayor varianza posible para todas las combinaciones de la forma de la ecuación (1), las cuales no están correlacionadas con $Y_{1}$. Es decir, $a_{2}$ se elige de modo que la varianza de $\mathbf{a}_{1}^{T} \mathbf{a}_{1}$ sea un máximo entre todas las combinaciones lineales de $\mathbf{X}$ que no están correlacionadas con la primera variable componente principal y tenga $\mathbf{a}_{2}^{T} \mathbf{a}_{2}=1$. En tal caso, 
dicho máximo es igual a $\lambda$, el segundo eigenvalor más grande de $\Sigma$, y que este máximo ocurre cuando $\mathbf{a}_{2}$ es un eigenvector de $\Sigma$ correspondiente al eigenvalor $\lambda_{2}$. De manera similar, pueden definirse las componentes principales restantes $\mathrm{Y}_{3}, \ldots, \mathrm{Y}_{\mathrm{p}}$. La j-ésima componente principal $(\mathrm{j}=3,4, \ldots, p)$ se expresa por $\mathbf{a}^{T} \mathbf{X}$ en donde $\mathbf{a}_{\mathrm{j}}$ se elige de modo que $\mathbf{a}_{j}^{T} \mathbf{a}_{j}=1$ y de forma que la varianza de $\mathbf{a}_{j}^{T} \mathbf{a}$, sea un máximo entre todas esas combinaciones lineales de $\mathbf{X}$ que no estén correlacionadas con las componentes principales restantes. De tal suerte, que este máximo es igual a $\lambda_{i}$, el j-ésimo eigenvalor más grande de $\Sigma$ y que satisface $\mathbf{a}_{j}^{T} \mathbf{a}_{j}=1$. Por ende, $\lambda_{1}{ }_{1} \geq \lambda_{2} \geq \ldots \geq \lambda_{p}$ denotan los eigenvalores ordenados de 2 y $\mathbf{a}_{1}, \mathbf{a}_{2}, \ldots, \mathbf{a}_{\mathrm{p}}$ denotan ${ }_{\mathrm{l}}^{\mathrm{p}}$ eigenvectores normalizados correspondientes.

Así, si denotamos por $A$ a la matriz de $p \times p$ de eigenvectores: $\bullet$ y al vector de $p \times 1$ de componentes principales por $\mathbf{Y}$. Entonces:

$$
\mathrm{Y}=A^{T} \mathrm{X}
$$

En este sentido, la matriz de covarianzas de $\mathbf{Y}$ se denotará por ? y está dada por

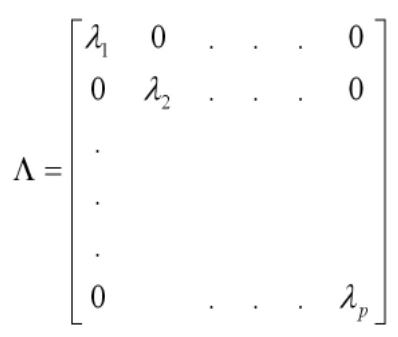

La matriz es diagonal debido a que los componentes se han elegido de manera que no estén correlacionados. Los eigenvalores pueden interpretarse como las respectivas varianzas de los distintos componentes. Si $\operatorname{tr}(\Sigma)=\sigma_{11}+\sigma_{22}+\ldots+\sigma_{\text {p. }}$. Por lo tanto $\operatorname{tr}(\Sigma)$, en cierto sentido, mide la variación total en las variables originales. Por su parte, la suma de las varianzas de los componentes está dada por

$$
\begin{gathered}
\sum_{i=1}^{p} \operatorname{Var}\left(Y_{i}\right)=\sum_{i=1}^{p} \lambda_{i}=\operatorname{tr}(\Lambda) \\
\mathrm{y} \\
\operatorname{tr}(\Lambda)=\operatorname{tr}(\Sigma)=\sum_{i=1}^{p} \operatorname{Var}\left(X_{i}\right)
\end{gathered}
$$

Con ello, se deduce que la suma de las varianzas de las variables originales y las de sus componentes principales son iguales. En otras palabras, la variación total explicada por las variables componentes principales es igual a la cantidad total de la variación medida por las variables originales. 
Por lo tanto, el i-ésimo componente principal explica una proporción $\lambda_{i} / \sum_{j=1}^{p} \lambda_{j}$ de la variación total en los datos originales. De esto se sigue, que los primeros m componentes explican una proporción $\sum_{j=1}^{m} \lambda_{j} / \sum_{j=1}^{p} \lambda_{j}$ de
la variación total.

En todo caso, la interpretación que surge del uso del tipo de variables como las del estudio, que corresponden con una correlación positiva, donde, el primer componente principal es una especie de promedio ponderado de las variables y puede considerarse como una medida de tamaño. De cualquier manera, las componentes principales tienen que ser transformadas en variables porcentuales que permiten dilucidar las distancias existentes entre una entidad a otra y en este sentido, estimar que el trabajo necesario, en combate a la pobreza tiene que ser arduo si se pretende alcanzar la cabeza de grupo; como es el caso de la delegación Benito Juárez. 